\title{
e-Phaïstos
}

e-Phaïstos

Revue d'histoire des techniques / Journal of the history

of technology

V-2 | 2016

Histoire des techniques en Afrique de l'Ouest

\section{Architecture et identités techniques au Cameroun}

Architecture and Technical Identities in Cameroon

\section{Rémy Dzou-Tsanga}

\section{(2) OpenEdition \\ Journals}

\section{Édition électronique}

URL : http://journals.openedition.org/ephaistos/5756

DOI : 10.4000/ephaistos.5756

ISSN : 2552-0741

\section{Éditeur}

IHMC - Institut d'histoire moderne et contemporaine (UMR 8066)

\section{Édition imprimée}

Date de publication : 15 juin 2016

Pagination : $36-48$

ISSN : 2262-7340

Référence électronique

Rémy Dzou-Tsanga, «Architecture et identités techniques au Cameroun », e-Phaïstos [En ligne], V-2 I

2016, mis en ligne le 15 mars 2020, consulté le 17 juin 2020. URL : http://journals.openedition.org/ ephaistos/5756 ; DOI : https://doi.org/10.4000/ephaistos.5756 


\title{
Architecture et identités techniques au Cameroun
}

\author{
Remy Dzou Tsanga \\ Ph.D, Chargé de cours \\ Université de Maroua \\ Faculté des lettres et sciences \\ humaines \\ Département d'histoire
}

\section{Introduction}

Situé en Afrique centrale à l'Extrême Nord Est du Golf de Guinée, le Cameroun s'étend du $9^{\mathrm{e}}$ au $16^{\mathrm{e}}$ degré de longitude Est et s'étire du $2^{\mathrm{e}}$ au $13^{\mathrm{e}}$ degré de latitude Nord ${ }^{1}$. L'espace géographique ainsi délimité se distingue par sa diversité écologique et ethnique $^{2}$. Le pays est le champ fertile d'une production artistique locale riche et variée. On peut citer l'artisanat et ses nombreuses filières telles que la céramique, la sculpture, la vannerie, la forge, la peausserie, la teinturerie, le tissage qui sont à l'origine d'un ensemble de productions artisanales. Dans ce registre figure en bonne place, l'architecture endogène ou vernaculaire. Sa phénoménologie permet de se rendre compte de l'importance qu'elle occupe dans la vie des peuples en tant qu'espace domestique, liturgique, commercial, funéraire, industriel et stratégique.

A l'évidence, la production architecturale couvre un vaste domaine de recherche qui attire la curiosité des explorateurs, des administrateurs coloniaux et des scientifiques. Parmi ceux-ci, nous citons Zenker3, Dominik4, Tessmann5, Begin6, A.M.D.Lebeuf 7 Mveng ${ }^{8}$, Laburthe-Tolra9, Christian Seignobos et F. Jamin ${ }^{10}$, W. Lauber ${ }^{11}$ et beaucoup d'autres. Les travaux de ces auteurs sont faits soit dans le cadre des monographies locales ou régionales, soit dans le contexte de l'étude des œuvres architecturales construites par les Européens au Cameroun. L'accent est mis sur la description formelle des structures qui semble être le paradigme d'identification des peuples. On parle de la case obus des Mousgoum, l'habitation ronde des Foulbé, les palais quadrangulaires des kotoko au Nord-Cameroun.

Le sud Cameroun est le domaine de prédilection des habitations en écorces d'arbres, de la maison carrée des Bamilékés et la hutte en forme de dôme des pygmées. Ces faciès architecturaux sont le reflet de la contrainte écologique. En réalité, le problème de la connaissance des techniques de construction se pose de manière effective. Au-delà de la diversité ethnique comment définir les identités techniques régionales à partir de l'analyse des œuvres architecturales? Le fait des identités techniques est-il vérifiable ? Ces questions d'intérêt scientifique avéré, permettent de poser au préalable, les fondements méthodologiques d'analyse des œuvres architecturales et de dégager les particularismes techniques en fonction des matériaux que livre le milieu écologique.

\section{Fondements méthodologiques d'analyse des techniques architecturales}

L'objet d'étude de cette recherche est l'architecture endogène. Celle-ci englobe des constructions de nature différente. Elles sont disséminées dans le pays. Les œuvres architecturales sont construites et utilisées par les populations locales avant le contact avec l'altérité le 12 juillet 1884 date de signature du traité germanodouala $^{12}$. Et, à partir de ce moment, on assiste au 
déclin des techniques de construction ancestrales alors en vigueur. Pendant la période coloniale 18841961 de nouvelles techniques sont introduites et connaissent une expansion dans le pays. Dans cette étude, la base méthodologique d'analyse des témoins architecturaux s'appuie sur l'exploitation des sources écrites, iconographiques, et des observations directes sur le terrain.

Les sources écrites regroupent pour l'essentiel la littérature spécialisée. La recherche documentaire s'est faite dans les bibliothèques du Ministère de la Recherche Scientifique et de l'Innovation (MINRESI), de l'Université de Yaoundé I, des centres culturels (allemand et français), et à l'Institut pour la Recherche et le Développement (I R D). Pendant cette étape de la recherche, les travaux spécifiques sur les Mousgoum ${ }^{13}$ et les Beti se sont avérés remarquables ${ }^{14}$. Mais cette littérature scientifique reste marquée par une lacune en ce qui concerne les techniques utilisées dans les différents espaces géographiques du pays. Aussi, avons-nous privilégié les observations sur le terrain, lesquelles sont complétées par l'iconographie. De ce point de vue, l'iconographie constitue une source d'informations importante car elle transmet une part de la mémoire des aspects de la culture matérielle qui ont physiquement disparu.

Dans les localités où la construction des cases endogènes a cessé, les prises de vue ont été faites sur des reconstitutions en particulier la case obus des Mousgoum, le corps de garde (abàà) et la cuisine de la femme (ndà) en pays béti. Ces reconstitutions sont examinées à l'effet, d'appréhender les modalités de construction. Il est question de procéder à la lecture des murs pour identifier les matériaux et le système d'appareillage utilisés. Ce travail est enrichi par les enquêtes ethnographiques. L'objectif poursuivi vise la collecte d'informations sur les matériaux de construction et le procédé de réalisation. Cette dernière étape de la recherche nous semble opportune par ce qu'elle facilite la définition des modèles locaux considérés comme des vecteurs de la communication d'identité sociale. Les données enregistrées sur le terrain permettent de dire que la production architecturale endogène est tributaire du milieu écologique.

\section{Production architecturale et contrainte écologique}

L'architecture vernaculaire est le produit de l'interaction entre l'homme et son environnement. Cependant, Il faut relever que les formes architecturales peuvent être influencées par des règles sociales, des croyances et des rites. Le déterminisme physique de l'architecture est remis en question par Rapoport ${ }^{15}$ qui pense que l'œuvre architecturale doit être appréhendée à l'intérieur de la relation entre l'environnement et la culture. La construction des œuvres architecturales répond aux besoins de l'homme de se protéger et de se défendre. Les expressions architecturales se sont ainsi diversifiées au fil des millénaires pour offrir plus de sécurité, de stabilité et de confort à l'homme. Au niveau historique, les traces de fondation de cases et les restes de poteaux se révèlent au Cameroun depuis le néolithique. La présence des villages est attestée au sud-Cameroun dans la région de Yaoundé et ses environs aux sites d'Obobogo, de Nkang et Ndindan dont l'occupation couvre la seconde moitié du premier millénaire et le premier millénaire $\mathrm{BC}$ d'après de Maret ${ }^{16}$ et C.Mbida ${ }^{17}$. Ailleurs dans la partie septentrionale, l'implantation humaine dans les monts Mandara est manifeste à travers de nombreux vestiges architecturaux qui sont relativement bien conservés notamment les DGB (di-gid-bay) qui désignent, en langue mafa, des «abandoned house platforms of the chief $\gg 18$. Toutefois, l'étude de ces structures en pierres sèches impose l'implication des architectes pour une harmonisation des concepts.

En effet, l'œuvre architecturale est le reflet partiel de l'environnement naturel dans lequel elle se trouve, et où l'homme expérimente les procédés de construction. L'espace bâti est de ce fait, 
l'expression des comportements humains (behaviours) comme le fait savoir Rapoport cité plus haut. Les rapports entre le groupe humain et son milieu naturel sont à l'origine des systèmes techniques. Dans cette étude, le Cameroun est divisé en trois zones écologiques. La partie méridionale qui s'étend du Sud de l'Adamaoua à la côte, regroupe sept régions administratives (Centre, Est, Ouest, Littoral, Nord Ouest, Sud-Ouest, Sud). La zone septentrionale couvre les régions de l'Adamaoua, du Nord et de l'Extrême Nord. Cette dernière région se trouve dans le domaine sahélien.

\section{Le Cameroun méridional, une civilisation du végétal}

La région est dominée par la forêt dense comme l'indiquent les études phytographiques' ${ }^{19}$. La savane occupe des espaces très réduits. La présence des îlots de savane dans le domaine forestier résulte des actions anthropiques notamment la pratique de l'agriculture itinérante sur brulis. Ces deux formations végétales sont des gisements de matières premières pour la production sculpturale et architecturale locales qui est une des spécificités de la région. Le végétal sert à fabriquer le mobilier domestique (notamment, lits, claies, peignes et louches en bois) et les vêtements en écorces (obom en langue ewondo).

Les peuples qui y habitent sont des pygmées considérés à tort ou à raison, comme étant, les premiers occupants de la forêt. A ce groupe primitif, s'ajoutent les Pahouins (Fang-Boulou-Bëti) ${ }^{20}$, les bamiléké, les bamoun, les sawa, les Maka, les tikar. Ces peuples, exceptés les pygmées, présentent une parenté linguistique et sont désignés par le terme "bantou "21. L'adaptation de ces peuples dans le milieu forestier a fait éclore une civilisation du végétal. Le végétal occupe une place centrale dans la vie des populations de la zone forestière. Il sert à nourrir les hommes et les animaux. Il constitue un gisement de produits pour la médecine traditionnelle comme le fait savoir Mve Belinga ${ }^{22}$.
On ne peut négliger le rôle d'interface qu'il joue entre le monde des vivants et celui des morts. Dans ce sens, l'arbre est le lieu d'inhumation des cadavres $^{23}$. Chez les Eton du Cameroun, le poteau central sculpté de la case de l'homme (l'abàà) a une dimension spirituelle. Ce poteau supporte le toit de la maison comme Dieu le fait avec la voûte céleste. Chez les Béti du Cameroun central par exemple, « le ficus du pays » (otombo nnam) est un arbre que l'on plante sur la tombe d'un grand chef où dans un village pour enterrer la hache de guerre entre les communautés en conflit. L'arbre planté est presque sacré comme le fait savoir Théodore Tsala ${ }^{24}$. Cette dimension religieuse du végétal s'observe aussi à l'Ouest du pays où les bamilékés en l'occurrence, délimitent et conservent un espace sylvicole appelé «forêt sacrée » destinée au chef et sa communauté.

En ce qui concerne l'architecture, de nombreuses espèces végétales sont recherchées et sélectionnées dans la forêt pour servir de matériaux de construction. Les essences sont choisies en fonction de leur capacité à résister à l'activité biologique et climatique. Parmi les bois qui sont sollicités on peut mentionner le Coula Edulis, Chlorophora Excelsa, Baillonella Toxisperma, Uapaca Guinnensis, Distemonanthus, Triplochiton Scleroxylon. Le bois sert à l'équarrissage des piquets et des poutres. Les écorces d'Ayous (Tripocliton Scleroxylon) couvrent les murs des cases. Cependant, deux patriarches interrogés à Ngoulmekong mentionnent l'usage d'écorces des essences telles que, ntui (Caloncoba GlaucaFlacourtiacées), Otùngui (Polyalthia Suaveolensannonacées), dùm (Ceiba Pantandrabombacacées) en langue ewondo 25 .

Il n'y a pas que du bois qui entre dans la construction: d'autres espèces végétales interviennent, c'est le cas du raphia (Raphia Vinifiera) qui se développe dans les marécages. Cette plante fournit les éléments suivants : folioles, nervures, et bambous. Ces matériaux servent à confectionner des nattes. Le raphia est également recherché pour la qualité du vin qu'il procure et les 
larves d' hanneton très appréciés par les populations qui en consomment.

Le tressage de la natte comporte plusieurs étapes. D’abord les feuilles fraîches sont arrachées des branches à la main ou à la machette. Ensuite, elles sont soigneusement emballés en paquets (ebag-zam ou mbom en langue ewondo) lesquels sont transportés à la tête jusqu'au site de construction. Ici, l'homme travaille seul ou en groupe. La natte se compose des folioles et deux lamelles de bambou (miwom).

Le procédé consiste à plier la feuille de raphia en deux sur les baguettes disposées parallèlement et à les épingler plusieurs fois sur toute la longueur de la natte qui atteint parfois $6 \mathrm{~m}$. Il ne suffit pas d'avoir les poteaux et les nattes pour construire. D'autres éléments sont nécessaires: pannes, traverses, poutre et lianes.

Les pannes et les chevrons sont faits de branches de raphia dépouillées de leurs feuilles et constituent les principaux matériaux de l'ossature de la charpente. Le problème que doivent résoudre les constructeurs est celui de la conception des murs qui assurent la protection des occupants. Il s'agit des matériaux qui entrent dans la confection du clayonnage. D'après le témoignage de Telesphore Mboudou ${ }^{26}$, les bâtisseurs ont recours à divers matériaux : branches de palmier à huile, de raphia et des troncs d'arbustes. L'autre difficulté est relative à la mise en place de la charpente.

Les techniciens utilisent les liens naturels notamment des lianes: mbikol, ongam, ebòdi, eka, ndin, a sag nlòn, kpekpà nlon edan mvin, tous des rotins dans l'ensemble comme le mentionne Laburthe-Tolra27. Mais, cet auteur ignore que tous les rotins ne sont pas utilisables dans la construction. En effet, ce «sont des palmiers grimpants épineux qui poussent dans les régions tropicales et semi-tropicales du vieux monde et sont exploités pour leurs tiges flexibles, appelées rotins ${ }^{28}$ » D'après les sources orales corroborées par les recherches de L.Defo ${ }^{29}$, les variétés de rotins employées dans la construction sont prioritairement le nlon (Eremospatha Macrocarpa) et le nding (calamus Deeratus). Ces deux variétés servent aussi à la confection des paniers, des chaises, et des hottes. Le choix est motivé par la flexibilité de ce matériau. Dans les localités où ces liens sont rares, on recourt aux cordes fabriquées à partir des fibres tirées des branches de palmiers à huile30. Le fil d'attache ou fil de fer fait son apparition pendant la période coloniale.

Les pygmées recherchent en priorité les feuilles d'une plante locale appelée akoué en langue Ewondo pour construire leurs huttes. Ses feuilles peuvent être transformées en récipients ou en emballages destinés au transport de l'eau et des produits. Dans un contexte d'économie traditionnelle marquée par l'absence du mobilier domestique moderne, la feuille d'akoué est importante pour ces populations.

Chez les Beti, la réalisation des habitations s'accompagne des pratiques magico religieuses qui structurent leur univers culturel. Les enquêtes ethnographiques menées dans les villages ne permettent pas d'éclairer suffisamment ces pratiques. Quoi qu'il en soit, Laburthe-Tolra s'est penché sur la question des rites de fondation des villages béti. L'auteur relate que «le père faisait creuser un trou et y déféquait, c'est dans ce trou qu'allait être planté le poteau central de l'abàà, plus tard richement décoré et auquel on suspendait les armes... »31. Cette observance a une signification dans le groupe social puisqu' «en accomplissant l'acte qui chez un homme ressemble le plus à un accouchement, le père a voulu fonder le village sur sa propre substance, sur la force qui émane et se détache de lui ... $\gg 32$. Le rituel ainsi accompli, marque le début effectif de la construction. Le technicien creuse des trous au le sol suivant un plan défini par le propriétaire de la case. De longs poteaux fourchus sont plantés aux extrémités de la case et supportent la poutre centrale qui forme le faîte33. Le dispositif est complété par les éléments 
de remplissage (poteaux) et l'ensemble constitue l'ossature du mur34.

L'abàà, case de l'homme ou corps de garde se distingue des autres cases du village par sa position topographique dominante. Ces dimensions sont importantes $10 \mathrm{~m}$ à $12 \mathrm{~m}$ de long sur $6 \mathrm{~m}$ à $8 \mathrm{~m}$ de large tandis que la ndà (cuisine) ne mesure que $4 \mathrm{~m}$ à $6 \mathrm{~m}$ de long sur $4 \mathrm{~m}$ à $5 \mathrm{~m}$ de large. L'abàà est le point de référence par rapport à l'organisation spatiale des structures qui composent l'habitat: tombe, fosse d'aisance (eduk), cuisine (ndà). La tombe est un élément structurant de l'habitat. Dans les représentations sociales des Beti, elle est considérée comme la demeure du défunt et occupe des emplacements précis au sein de la zone d'habitation.

Les sépultures des adultes se localisent au milieu de la cour. Parfois, elles occupent le nlo ndà qui signifie tête de la maison en langue ewondo. Cet espace se localise au niveau des pignons. En revanche, les nourrissons sont enterrés sous la véranda à proximité de la chambre à coucher (etun ndà) des parents car, l'enfant décédé doit renaitre. Dans le cas du suicide, la mort s'apparente à la souillure et à la honte, le cadavre est inhumé au lieu du décès. On peut comprendre l'intérêt que revête le corps de garde en tant qu'espace symbolique où l'homme, propriétaire et gardien du village, doit être puissant, force qu'il tient de sa case. Une fois l'implantation achevée, le technicien s'attaque à la confection de la charpente.

\section{La charpente}

La charpente est l'ossature du toit comme du bâtiment sur poteaux. Dans le sud-Cameroun, les toits de maison sont à deux versants ou de forme conique dans les grassfields. Ces deux types de charpente présentent une forte inclinaison qui facilite l'écoulement rapide des eaux et évitent le problème d'étanchéité. Les éléments qui forment la charpente sont, entre autres, les pannes, les chevrons en bambou et les liens (lianes). Le procédé
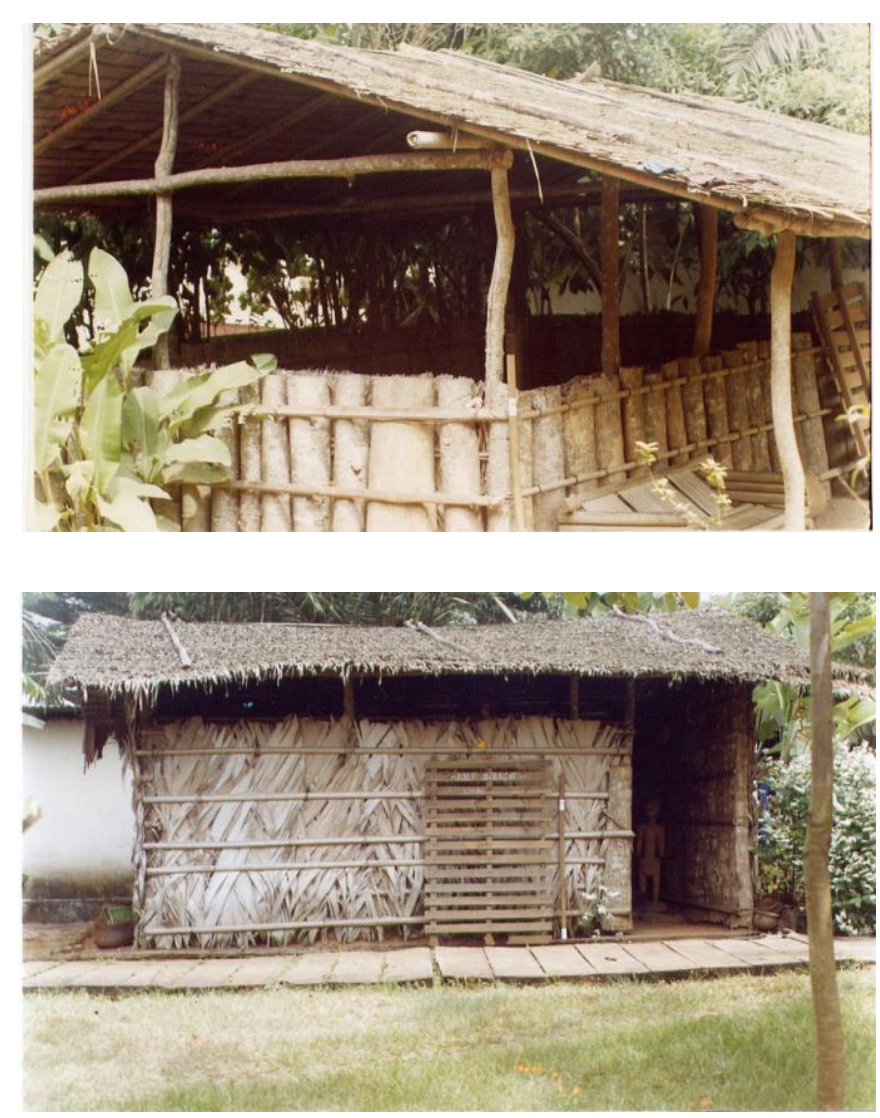

Figure 1 : reconstitution des cases en écorces. En haut, Case de l'homme (l'abàà) Elig Essono (Yaoundé) Photo DZOU TSANGA, le 25/o5/2013. En bas, Case de la femme (ndà) Elig Essono (Yaoundé) Photo DZOU TSANGA, le 25/o5/2013

de montage est l'assemblage par ligature. Pour les toits à deux versants, les chevrons sont disposés en $\mathrm{V}$ renversé sur la poutre centrale qui relie les deux pignons. Les pannes sont posées horizontalement sur les chevrons. L'ensemble est solidement fixé par les lianes. Au sujet des toits de forme conique, la charpente est faite de perches qui se joignent en bouquet au niveau du faîte. Les anneaux renforcent la structure. Cette technique est répandue dans la 
partie septentrionale du pays. Les matériaux servants de couverture sont variables. La natte de raphia et le chaume (Imperata Cylindrica) sont utilisés. Parfois, on a recours aux branches de palmier ou aux feuilles de bananier pour couvrir les installations sommaires. A propos des toits en nattes de raphia, le principe consiste à les ajuster comme des écailles de poisson du faîtage au bord et à les fixer avec des lianes. Une fois la toiture achevée, on procède à la confection des murs.

Les murs sont composés d'une armature porteuse et du revêtement. L'armature du mur est le «squelette» de la case. Elle est faite de poteaux en bois durs que l'on fixe solidement au sol pour garantir la stabilité et la solidité de la maison. Quant au revêtement, il est fait de plaques rectangulaires d'écorces d'arbres (bivin en langue Ewondo) d'où le nom (ndà bivin) qui signifie littéralement en français, maison des écorces. Les éléments sont disposés verticalement sur l'armature porteuse. Toutefois, le constructeur peut utiliser les branches de palmier à huile (Elæis guinéennsis). Celles-ci sont soigneusement dressées de manière à couvrir les interstices. A l'Ouest du pays, les murs sont en bambou. Dans l'ensemble, la technique employée pour construire les murs est l'assemblage par ligature.

\section{Le sol}

Si le sol de la case présente un relief accidenté, celui-ci subit un traitement. L'opération consiste à le remuer avec une houe tout en y aspergeant de l'eau. La surface du sol est recouverte de feuilles de bananier fraîches. Ce traitement peut varier. Le plus souvent, on incruste des noix de palme au sol pour des mobiles d'ordre esthétique selon l'aveu de Thérèse Fouda35.

\section{Les ouvertures}

Les ouvertures servent de voies d'entrée ou de sortie (portes) et d'aération (fenêtres). Les cuisines, espaces d'activités domestiques et de pratiques rituelles, obligent les Fang, Boulou et Beti à supprimer les fenêtres dans ces structures. Dans la majorité des cas, les portes sont faites de plaques d'écorces d'arbres (bivin) fixées sur deux barres par des lianes ${ }^{6}$. Dans l'abàà, les fenêtres sont des baies que l'on couvre avec les écorces (bivin) 37 .

Les producteurs des œuvres architecturales utilisent plusieurs outils même si l'outillage du bâtisseur est sommaire. Machette, hache, plantoir, houe sont essentiels. Ces outils ont des usages précis. La machette et la hache sont utilisées en priorité pour l'abattage et l'équarrissage du bois. Le plantoir sert à creuser les trous des poteaux. La houe sert à remuer le sol de l'intérieur des cases. Si la culture technique du végétal est largement répandue au sud-Cameroun, en revanche, celle de la terre domine la partie septentrionale.

\section{Le Cameroun septentrional, une civilisation de la terre cuite et crue}

Dans les régions de l'Adamaoua et du Nord, la savane constitue la formation végétale dominante ${ }^{38}$. Par contre la région de l'Extrême-Nord se trouve en pleine zone sahélienne même si les îlots de savanes existent à certains endroits. Dans cet espace géographique, le travail de la terre crue ou cuite est développé. En plus des activités agricoles saisonnières, la production architecturale est importante et présente plusieurs faciès.

Depuis des millénaires, le façonnage de la poterie fournit après la cuisson, la céramique (vases, jarres, assiettes, foyers, pots, objets de décoration, statuettes). Ces objets sont utilisés comme du mobilier domestique ou comme des objets rituels. La poterie est un artisanat essentiellement pratiqué par les femmes dans cette région. Les consommateurs se ravitaillent chez les artisanes et les grossistes dans les ateliers et les marchés. Parfois, ils les reçoivent sous forme de cadeaux. La production et l'utilisation de la céramique sont des éléments caractéristiques de la civilisation de la terre cuite des Sao dont le rayonnement est connu aux abords du lac Tchad 
selon Jean-Paul Lebeuf39. En parlant de la culture matérielle du Cameroun septentrional, il y a cette nécessité d'inclure la civilisation de la terre crue qui répond au besoin de s'adapter dans une région qui offre des matériaux de construction spécifiques. Deux particularités se dégagent lorsqu'on observe le bâti de cette partie du pays.

Dans les monts Mandara, le relief présente de nombreux massifs et inselbergs qui hébergent plusieurs groupes ethniques en majorité kirdi. Les témoins architecturaux observés montrent que, les populations optent pour deux solutions pour construire leurs habitations. Ils choisissent soit de faire uniquement la base du mur en pierres et le reste en torchis, soit d'élever toute la partie verticale en moellons. Pour ce dernier cas, la technique utilisée est l'appareillage qui, d'après Jean Luc François, consiste à la définition du principe de taille des pierres et dans leur agencement 40 . Les habitations traditionnelles, comme on peut le voir sur le terrain, rendent compte du procédé mis en place. Dans les montagnes, l'appareil irrégulier prédomine. Il se caractérise par l'usage des pierres plus ou moins équarries ${ }^{41}$. La pierre stabilise les structures porteuses et corrige la topographie du sol. Dans l'ensemble, la construction des œuvres architecturales affectionne les formes rondes. Le contact des montagnards avec l'administration coloniale au début du XXe siècle est à l'origine de la mutation des formes architecturales que l'on observe dans cette partie du pays. Désormais, la tendance est aux cases quadrangulaires couvertes de tôles ondulées. La nouvelle maison devient un élément de réussite ou d'hiérarchisation sociale.

En général, les toits des cases sont en majorité de forme conique et couverts de séko. Cette espèce végétale ne s'accommode pas de la grande chaleur qui sévit dans la région en saison sèche. Elle se développe sur les pentes des collines et dans les plaines. Son usage est répandu dans la partie septentrionale du Cameroun. Les techniciens s'approvisionnent auprès des vendeurs de nattes de séko. Ces derniers sont des cultivateurs qui abandonnent les activités agricoles en saison sèche pour se livrer à la collecte et à la vente de la paille. Selon nos observations, les bâtisseurs utilisent fréquemment les tiges de mil pour confectionner les toits. Ailleurs, dans l'Adamaoua et dans le Nord, la savane fournit le chaume (Imperata cylindrica) qui couvre les toits.

L'importance historique des plaines du Nord n'est pas liée uniquement aux activités agricoles piscicoles et pastorales. Elles sont davantage connues par la production architecturale qui est manifeste. On y retrouve, en marge des cases traditionnelles rondes habituelles, deux architectures singulières dont les symboles significatifs sont la case obus et la maison en terre des kotoko. Dans ces espaces, deux morphologies coexistent. Prépondérance de la forme ovoïdale chez les Mousgoum et de la forme quadrangulaire chez le kotoko. Exception faite de ces constructions entièrement en terre, les habitants des plaines privilégient le torchis pour revêtir les parois des cases et des greniers.

Le torchis et le pisé sont des techniques de construction anciennes répandues dans les régions où le bois manque. Elles furent expérimentées aussi bien en Europe, en Amérique, en Asie qu'en Afrique comme le souligne Leroi-Gourhan ${ }^{42}$. Au Cameroun, le torchis est connu dans les zones soudano sahéliennes. Dans l'état actuel des recherches et, en l'absence des données chronologiques, il n'est pas possible de situer l'origine de la technique. Selon les observations de terrain, les bâtisseurs prennent la terre à proximité du site de construction. Le plus souvent, ils récupèrent les murs de cases délabrées et les concassent. La terre obtenue est mélangée aux végétaux ou du crottin de chèvres. Le tout est dégraissé avec du sable. La terre hétérogène est arrosée avec de l'eau puis laissée au repos pendant des heures.

Les espèces végétales fréquemment employées dans la préparation du torchis sont le séko, les tiges de riz. La terre enrichie des débris de paille est pétrie à un ou à deux pieds jusqu'à obtenir la 
consistance voulue. C'est ce mélange homogène qu'on appelle torchis. La préparation du matériau dure environ une trentaine de minutes et l'activité exige un effort physique important. Toutefois, la composition du torchis varie selon les peuples, les lieux, les recettes, et les ressources.

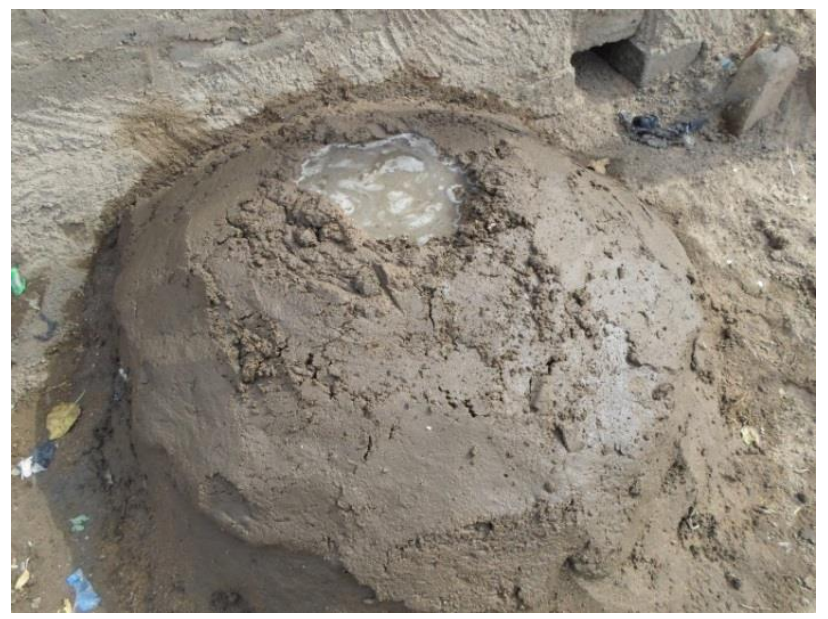

Figure 2 : préparation du torchis dans un chantier de construction à Dogba, Extrême-Nord, Photo DZOU TSANGA, le 20/O1/2014.

Chez les kotoko de Makari par exemple, Lebeuf mentionne les ajouts tels que des petits os, des coquillages et surtout des tessons de poterie ${ }^{43}$. Cela équivaut à adjoindre un granulat pour obtenir une structure se rapprochant du béton, ayant pour effet d'améliorer le drainage-séchage du matériau et en augmenter la résistance et la dureté. En sillonnant le pays Mousgoum, on se rend compte que la case obus a disparu. Sa connaissance n'est rendue possible que par le biais de l'étude menée par Seignoboss et Jamin qui reste une référence. On ne saurait la reprendre pour éviter les redites. A propos du torchis qu'ils appellent «le matériau principal ", celui-ci est un mélange composé d'herbes suksukiy, d'excréments de chèvre et de terre argileuse44». Le crottin de chèvre fait office de liant. Ces habitations rondes de 3 à 5 mètres ne comportent qu'une seule ouverture: la porte d'entrée et de sortie.

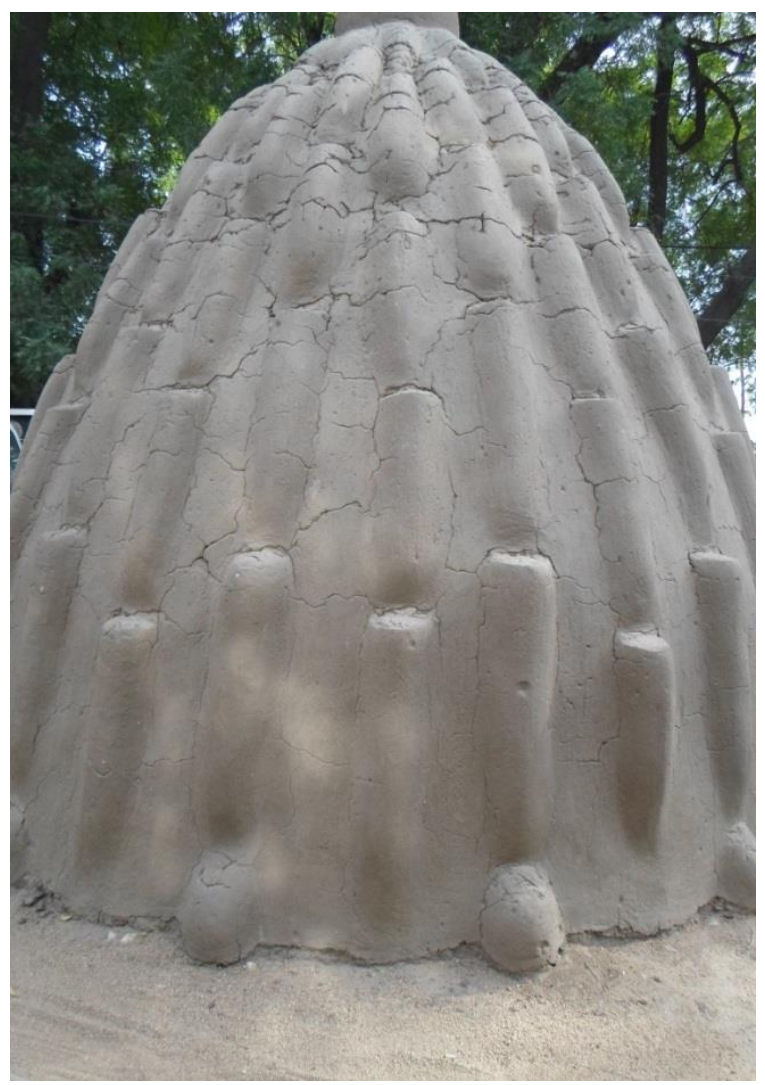

Figure 3 : Reconstitution de modèle de case Mousgoum, Photo DZOU TSANGA, le 23/o1/2014

Lorsqu'il s'agit de construire les habitations, chaque bâtisseur planifie son travail de construction de préférence en saison sèche. En matinée, il prépare la quantité de torchis qu'il va utiliser durant la journée. Une fois le lieu d'implantation délimité 
avec la houe, le technicien coupe manuellement le torchis en mottes. Le matériau est étalé sur les contours du plan et forme la couche de base de $15 \mathrm{~cm}$ de hauteur. Le torchis est déposé par couches successives de bas en haut. L'opération est répétée jusqu'à la hauteur souhaitée du mur qui ne dépasse pas 3mètres généralement. Lorsque l'élévation de la paroi qui dure sept jours en moyenne est terminée, on amorce la confection du toit de forme conique. L'ossature du toit est composée de perches assemblées par ligature et consolidées par des anneaux. Le toit est couvert de chaume et forme un bouquet au sommet. L'intérieur est souvent bien aménagé et couvert de sable. Toutefois une étude rétrospective permet de saisir le fait de l'identité technique dans l'art de bâtir dans l'espace géographique. Cette réalité est perceptible lorsqu'on explore les œuvres architecturales construites dans les différentes zones écologiques définies. Au sudCameroun, la case des bamiléké et la maison des béti par exemple, sont le fait d'assemblages par ligature. En revanche au nord-Cameroun les cases en torchis dominent.

\section{Le fait des identités techniques dans le cadre écologique du Cameroun}

L'étude amorcée dans cet article est centré sur a période précoloniale. La recherche porte sur l'architecture traditionnelle synonyme de précarité et d'ingéniosité de l'homme qui a su exploiter les éléments que la nature lui a offert. A priori, on a laissé entendre que l'Afrique subsaharienne n'avait pas d'architecture. Il était difficile d'admettre que l'architecture traditionnelle camerounaise soit porteuse des identités techniques à cause de sa vulnérabilité et de sa fragilité. A observer le paysage architectural local, on a l'impression que chaque groupe ethnique a son architecture particulière. C'est par les formes apparentes que l'on attribue les types architecturaux à un peuple ou à une région donnée. Ce qu'on oublie est que, la technique s'élabore en fonction des matières premières disponibles. Il devient possible d'appliquer certaines techniques particulières à un type de matériaux présents dans un milieu précis. De ce fait, il importe de dégager des singularités techniques en fonction des matériaux et des procédés de réalisation. Il faut noter que dans la zone forestière, l'assemblage par ligature des éléments s'impose pour construire les habitations en écorces d'arbres. De même, les somptueux palais des chefferies traditionnelles en pays bamiléké sont réalisés avec le même procédé et s'imposent formellement comme une originalité de l'architecture camerounaise dans le monde.

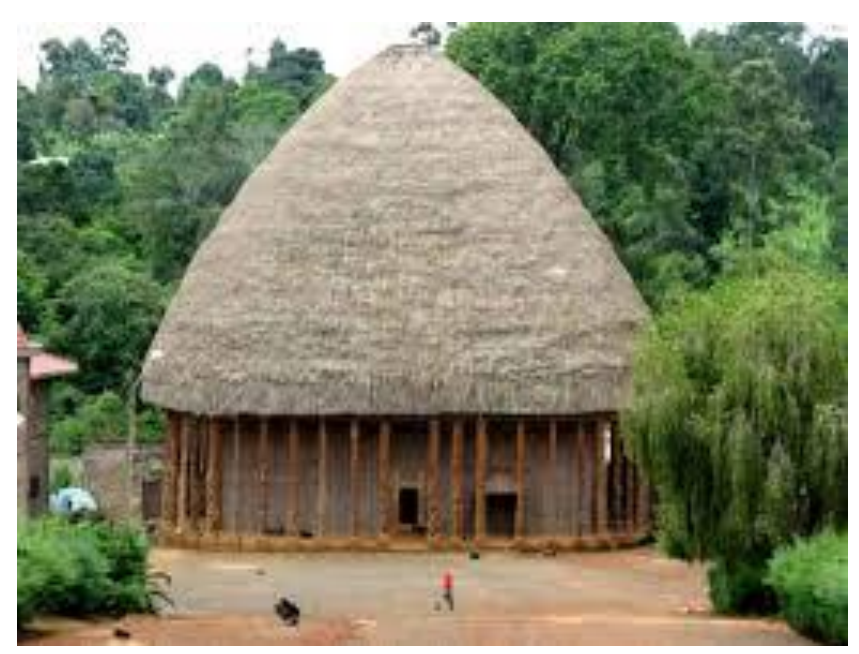

Figure 4 : exemple de modèle de case traditionnelle en vigueur chez les bamiléké. Source : Testa Olivier, o8/04/2011

Que dire de la hutte des pygmées où les feuilles sont soigneusement ajustées en écaille de poisson pour facilité l'écoulement des eaux de pluie? Les huttes sont groupées et forment des campements provisoires. Le matériau utilisé est biodégradable; et le renouvellement des huttes se fait régulièrement. 


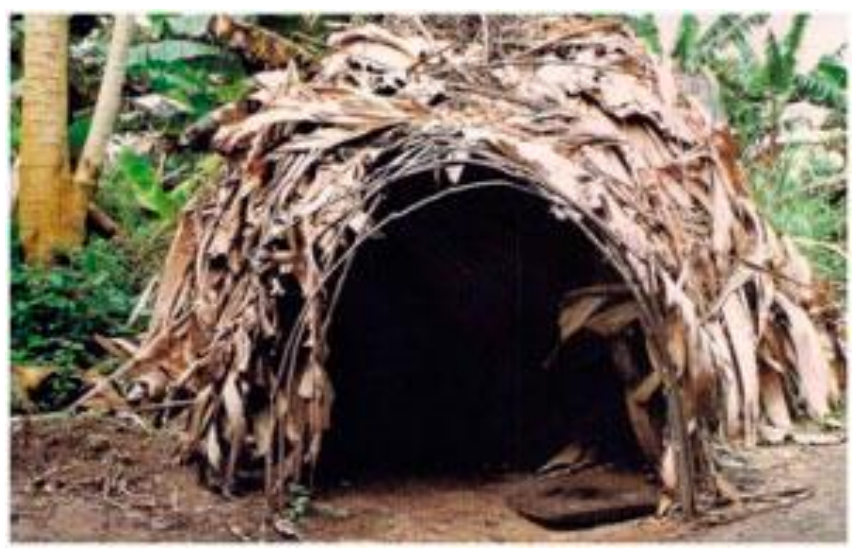

Figure 5 : Un exemple de hutte des pygmées. Source : http://mastervoyages.com/pygmees.php, consulté le $12 / 08 / 2014$

Le torchis dans la partie septentrionale du pays contribue au façonnage de l'une des plus belles architectures traditionnelles du monde, la case obus des Mousgoum et les palais kotoko. Ces productions sont venues enrichir le patrimoine immobilier mondial. Cette diversité des techniques architecturales constitue en effet, une particularité de l'architecture locale.

\section{Conclusion}

L'analyse des types architecturaux caractéristiques des principales zones écologiques mentionnées nous autorise à dire que, les identités techniques sont un fait. On peut les appréhender aussi bien dans la partie méridionale que septentrionale. Les civilisations de la terre et du végétal ont largement contribués à l'élaboration des procédés diversifiés de construction. La culture à travers les comportements humains a fortement marqué l'environnement bâti. De ce fait, elle a fait éclore des modèles architecturaux dont on peut s'enorgueillir.
Les témoins de l'architecture étudiés sont entrain de disparaître. De ce fait, toute l'histoire de la construction traditionnelle risque de s'évanouir dans l'oubli. La rencontre avec l'altérité et notamment la civilisation occidentale a largement contribué à ce phénomène. Une telle étude facilite la conservation du patrimoine architectural qui reste un facteur important pour le développement du tourisme culturel.

1 Aaron Suh NEBA, Géographie moderne de la république unie du Cameroun, Camden(USA), Editions Neba, 1987, p.1

2 DUGAST I, Inventaire ethnique du sud-Cameroun, Cameroun, Mémoire de l'IFAN, série population, $\mathrm{n}^{\circ}{ }^{\circ}$, 1949, Mveng E., Histoire du Cameroun, Paris, Présence Africaine, 1963 et Seignobos, Christian et Iyébi Mandjeck, Olivier, Atlas de la province de l'extrême nord, Paris, IRD, 2000

3 ZENKER Georg traduit par LABURTHE- TOLRA sous le titre, Yaoundé d'après Zenker 1895, In Annales de la Faculté des lettres et sciences humaines de l'Université de Yaoundé, $\mathrm{n}^{\circ} 1,1970$

4 DOMINIK Hans, Kamerun: sechs kriegs-und friedensjahre in deutschen topen, Berlin Erst Siegfried Mittler§ sohn, in- $8^{\circ}, 1901$

$5 \quad$ TESSMANN Gunter, Die pangwe,völkerkuldliche
monographie
negerstammen,Lübeck,1913
negerstammen, Lübeck,1913

6 BEGIN J.P. L'habitat au sud Cameroun: présentation des principaux types d'habitat .Essai d'adaptation aux problèmes actuels, Paris, ORSTOM, 1952

7 LEBEUF AMD, Les principautés kotoko. Essai sur le caractère sacré de l'autorité, Paris CNRS, 1969

8 MVENG E. L'art et l'artisanat africains, Yaoundé, Editions CLE, 1980

9 LABURTHE-TOLRA, Philippe, les seigneurs de la forêt, paris, publications de la Sorbonne, 1981

10 SEIGNOBOS Christian et Jamin Francois, La case obus. Histoire et reconstitution, Marseille, Editions Parenthèses, 2003

11 LAUBER Wolfgang (dir.), Architecture allemande au Cameroun de 1884 à 1914, Stuttgart, Editions Karl krämer, 1988

12 MVENG E., opcit, 1963 et Ngoh J V, Cameroun 1885-1985 cent ans d'histoire, Yaoundé, CEPER, 1990 
13 C.SEIGNOBOS et F.; JAMIN, opcit, 2003

14 LABURTHE-TOLRA, Ph, opcit, 1981 et Dzou Tsanga Rémy « Dynamique de l'architecture dans la lékié entre 1884 et 1960: approches historique et archéologique.» Thèse de Doctorat Ph.D en archéologie, Université de Yaoundé I, 2013

15 RAPOPORT, Amos, 1969b, House form and culture, PrinticeHall, Englewood Cliffs,N.J.Toronto, Printice-Hall of Canada.

16 DE MARET,P « Sédentarisation, agriculture et métallurgie du Sud-cameroun :synthèse des recherches depuis 1978.In Essomba J M(éd.) L'archéologie au Cameroun. Paris, Karthala, 1992, pp.247-262

17 Mbida MINDZIE C, «L'émergence des communautés villagéoises au cameroun méridional : étude archéologique des sites de Nkang et de Ndidan ».Thèse de Doctorat Ph.D (non publiée) Université Libres de Bruxelles,1996

18 Andrea Dawn RICHARDSON, « A eye of the chief on top archaeological investigation of DGB sites Northern Cameroon». Master of Arts thesis, University of Calgary,2004, p.2

19 LETOUZET R Etude phytogéographique du Cameroun. Encyclopédie biologique 69 .Paris, Editions P Lechevalier, 1968

20 ALEXANDRE Pierre et BINET J. Le groupe dit Pahouin (Fang Boulou-Bëti), collection des Monographies Ethnologiques africaines publiées sous le patronage de l'Institut International Africain, in- $8^{\circ}$, une carte, Paris, PUF, 1958

21 MARCHAL NASSE Colette, « Les bantou entité linguistique ». In aux origines de l'Afrique centrale; Lanfranchi $\mathrm{R}$ et Clist $\mathrm{B}$ (éds), Libreville, CICIBA, 1991, pp.135-143

22 MVE BELINGA Jeannot, «La médecine traditionnelle et l'évolution de la santé au Cameroun: le cas des Fangs Béti Boulou, 1924-2003 " Thèse de Doctorat Ph.D (non publiée) en histoire, Université de Yaoundé I, 2012

23 DZOU TSANGA R., opcit, 2013, p.64

24 VINCENT Jeanne-Françoise et BOUQUIAUX Luc (éds), Mille et un proverbes Béti recueillies par Théodore Tsala. Publié avec le concours du centre national de la recherche scientifique et de l'office de la recherche scientifique et technique d'outreMer, Louvain, 1986

25 Entretien avec Mvondo Melingui, 73 ans, planteur, Ngoulmekong, le 08/08/2007

26 Entretien avec Telesphore Mboudou, 74 ans, planteur, Ngoulmekong, le 07/08/2007)
27 LABURTHE-TOLRA. opcit, 1981, p. 265

28 DEFO Louis, Le rotin la forêt et les homes. Exploitation d'un produit forestier non ligneux au sud cameroun et perspective de développement durable. Netherland, Institute of environnemental sciences Leiden University ,2005 p.49

29 DEFO Louis. opcit p. 92

30 Entretien avec Mvondo Melingui, 73 ans, planteur, Ngoulmekong, le 08/08/2007.

31 LABURTHE-TOLRA, opcit, 1981, p.206

32 LABURTHE-TOLRA, opcit, 1981, p.206

33 Entretien avec Mvondo Melingui, 73 ans, planteur, Ngoulmekong, le 08/08/2007

34 Idem.

35 Entretien avec Thérèse Fouda, 60 ans environ, pharmacienne, Yaoundé, le 21/01/2004

36 Entretien avec Mvondo Melingui, 73 ans, planteur, Ngoulmekong, le 08/08/2007

37 Idem

38 Aaron Suh NEBA Opcit,1987, p.32

39 LEBEUF Jean Paul. Archéologie tchadienne: les Sao du Cameroun et du Tchad. Paris, Hermann, 1962

40 FRANÇOIS Jean-Luc, « Les fondations des constructions monumentales. Evolution au cours des siècles. "In méthodes et initiations d'histoire et d'archéologie. Racinet Philippe et Schwerdroffer Joël (coord.) Paris, Editions du Temps, 2005, p.259

41 FRANÇOIS Jean-Luc. Opcit, 2005, p.260

42 LEROI-GOURHAN A. Milieu et technique, Paris, Albin Michel, 1973, pp.233-240

43 LEBEUF A.M.D. Opcit. 1969, p.84

44 SEIGNOBOS Christian et JAMIN Fabien, Opcit, 2003, p.152 


\section{Bibliographie}

AARON SUH NEBA, Géographie moderne de la république unie du Cameroun, Camden(USA), Editions Neba, 1987, p.1

ALEXANDRE Pierre et BINET J., Le groupe dit Pahouin (Fang Boulou-Bëti), collection des Monographies Ethnologiques africaines publiées sous le patronage de l'Institut International Africain, in- $8^{\circ}$, une carte, Paris, PUF, 1958

BELA Cyrille., « Les expressions sculpturales au Sud-Cameroun: le cas du pays Béti » Thèse de Doctorat Ph.D (non publiée) en histoire de l'art, Université de Yaoundé I, 2007

BEGIN J.P., L'habitat au sud Cameroun: présentation des principaux types d'habitat .Essai d'adaptation aux problèmes actuels, Paris, ORSTOM, 1952

DEFO Louis, Le rotin la forêt et les hommes. Exploitation d'un produit forestier non ligneux au sud Cameroun et perspective de développement durable. Netherland, institute of environnemental sciences Leiden University, 2005

DE MARET P., « Sédentarisation, agriculture et métallurgie du Sud-Cameroun: synthèse des recherches depuis 1978.In Essomba J M (éd.) L'archéologie au Cameroun. Paris, Karthala, 1992, pp.247-262

DOMINIK Hans, Kamerun: sechs kriegs-und friedensjahre in deutschen topen, Berlin Erst Siegfried Mittler§ sohn, in- $8^{\circ}, 1901$

DUGAST IDELETTE, Inventaire ethnique $d u$ sud-Cameroun, Cameroun, Mémoire de l'IFAN, série population, $\mathrm{n}^{\circ} 1,1949$

DZOU TSANGA Rémy, «Dynamique de l'architecture dans la Lékié entre 1884 et 1960 : approches historique et archéologique ».Thèse de Doctorat Ph.D (non publiée) en archéologie, Université de Yaoundé I, 2013
FRANÇOIS Jean Luc. " Les fondations des constructions monumentales. Evolution au cours des siècles. »In méthodes et initiations d'histoire et d'archéologie, Racinet Philippe et Schwerdroffer Joël (coord.) Paris, Editions du Temps, 2005, pp.25-264

LABURTHE-TOLRA Philippe, Les seigneurs de la forêt, paris, publications de la Sorbonne, 1981

LAUBER Wolfgang (dir.), Architecture allemande au Cameroun de 1884 à 1914, Stuttgart, Editions Karl krämer, 1988

LEBEUF AMD, Les principautés kotoko. Essai sur le caractère sacré de l'autorité, Paris CNRS, 1969

LEBEUF Jean Paul. Archéologie tchadienne: les Sao du Cameroun et du Tchad. Paris, Hermann, 1962

LEROI-GOURHANA., Milieu et technique, Paris, Albin Michel, 1973, pp.233-240

LETOUZET R., Etude phytogéographique du Cameroun. Encyclopédie biologique 69 .Paris, Editions P Le chevalier, 1968

MARCHAL NASSE Colette, « Les bantou Entité linguistique ».In aux origines de l'Afrique centrale; Lanfranchi $\mathrm{R}$ et Clist $\mathrm{B}$ (éds), Libreville, CICIBA, 1991, pp.135-143

Mbida MINDZIE Christophe, "L'émergence des communautés villageoises au Cameroun méridional : étude archéologique des sites de Nkang et de Ndidan ».Thèse de Doctorat Ph.D (non publiée) Université Libres de Bruxelles, 1996

MVE BELINGA Jeannot, "Evolution de la médecine traditionnelle au Cameroun » Thèse de Doctorat Ph.D (non publiée) en histoire, Université de Yaoundé I, 2012

Mveng Engelbert, Histoire du Cameroun, Paris, Présence Africaine, 1963 
L'art et l'artisanat africains, Yaoundé, Editions CLE, 1980

NGOH J. V., Cameroun 1885-1985 cent ans d'histoire, Yaoundé, CEPER, 1990

VINCENT Jeanne -Françoise et BOUQUIAUX Luc (éds), Mille et un proverbes Beti recueillies par Théodore Tsala. Publié avec le concours du centre national de la recherche scientifique et de l'office de la recherche scientifique et technique d'outre-mer, Louvain, 1986

RAPOPORT, AMOS, 1969b, House form and culture, Printice-Hall, Englewood Cliffs,N.J.Toronto, Printice-Hall of Canada

RICHARDSON Andrea Dawn, " A eye of the chief on top archaeological investigation of DGB sites Northern Cameroon». Master of Arts thesis, university of Calgary, 2004

SEIGNOBOS, Christian et JAMIN François, $L a$ case obus. Histoire et reconstitution, Marseille, Editions Parenthèses, 2003

SEIGNOBOS, Christian et IYEBI Mandjeck, Olivier, Atlas de la province de l'Extrême Nord, Paris, IRD, 2000

TESSMANN Gunter, Die pangwe,völkerkuldliche monographie eines west afrikanischen negerstammen,Lübeck,1913

ZENKER Georg, traduit par LABURTHETOLRA sous le titre, Yaoundé d'après Zenker 1895, In Annales de la Faculté des lettres et sciences humaines de l'Université de Yaoundé, $\mathrm{n}^{\circ} \mathbf{1 , 1 9 7 0}$

\section{Source orales}

Mvondo Melingui, 73 ans, planteur, Ngoulmekong, le 08/08/2007

Thérèse Fouda, 60 ans environ, pharmacienne, Yaoundé, le 21/01/2004
Telesphore Mboudou, 74 ans, planteur, Ngoulmekong, le 07/08/2007) 